\title{
An 8.8-magnitude earthquake in Chile: immediate impact on perioperative services
}

\author{
Alejandro González, MD · Silvana Cavallieri, MD
}

Received: 8 November 2011/Accepted: 14 December 2011/Published online: 21 December 2011

(C) Canadian Anesthesiologists' Society 2011

\section{To the Editor,}

Some countries are exposed to severe natural disasters. We comment here on our experience facing this type of disaster in Chile, a country with one of the highest rates of earthquakes on the planet.

At dawn on Saturday February 27, 2010, an earthquake with a magnitude of 8.8 on the Richter scale shook the central-south zone of Chile, and minutes later, a tsunami flooded the coastal towns. This was the sixth largest earthquake on record. Five hundred twenty-six people died, housing and highways were destroyed, several hospitals were damaged, and all basic services failed. The damaged area extended over $115,000 \mathrm{~km}^{2}$ and encompassed a population of more than 4.8 million inhabitants.

The Chilean Society of Anesthesiology decided to review the experience of those working in the damaged area in order to implement recommendations for a disaster workflow plan and, hopefully, to assist other localities at risk for similar disasters. In May 2010, a brief 22-question survey was sent to anesthesiology department heads at the hospitals in the earthquake stricken region to assess the earthquake's immediate impact on hospitals, operating rooms (ORs), and other anesthetic work areas.

We sent 21 surveys and received 20 replies from 20 hospitals located in different towns in the region. One

A. González, MD ( $₫)$

Facultad de Medicina, Pontificia Universidad Católica de Chile,

Santiago, Chile

e-mail: gonzalea@med.puc.cl

\section{A. González, MD}

Sociedad de Anestesiología de Chile, Santiago, Chile

S. Cavallieri, MD

Hospital de Niños Luis Calvo Mackenna, Santiago, Chile hundred ten anesthesiologists worked in those hospitals, and 17 of them were at work on the morning of the earthquake. Thirteen surgeries were in progress at the time, and no patients or anesthesiologists died or were injured. Those hospitals lost $35 \%$ of their beds and $69.3 \%$ of their OR capacity. Immediately following the earthquake, the anesthesiologists on duty led and/or collaborated in patient evacuation and in saving equipment in surgical and other areas of the hospital. They carried out these tasks in the dark, with no elevators (due to a power failure), and while being subjected to frequent aftershocks. Sixty-five patients had surgery on the day (a Saturday) of the earthquake, and 242 injured people had surgery during the first week after the event. Those surgeries were performed in non-damaged ORs and in hospitals providing emergency services.

None of the damaged hospitals collapsed; nevertheless, partial or total evacuation occurred in 15 hospitals due to identified structural risks. Damage to infrastructure, interruptions to water and electricity supply, and failure in telephone communications were the main problems faced by the anesthesiologists in those hospitals. No centre reported interruption of oxygen supply or disruption of drug or equipment supplies. The anesthesiologists reported feelings of fear and helplessness despite most respondents affirming they were able to resolve the initial emergency and healthcare-related demands during the first week after the event. Once the damage to infrastructure and staffing requirements were assessed, anesthesiologists began to request specific assistance from other health institutions in the region that were not impacted by the earthquake. Although contingency plans were in place and the local anesthesiologists could handle the immediate crisis, an earthquake of this magnitude went well beyond our usual level of emergency preparedness. Nevertheless, some lessons were learned on how to face such an emergency: 
- Each hospital must have a response plan with welldefined roles, clear patient evacuation procedures, and periodic staff training. Safety areas and evacuation routes should be clearly indicated.

- Water and electricity backup supply systems should be available.

- Walkie-talkies or satellite telephones must be available in case of failure of routine telephone communications.

- One anesthesiologist should be designated to organize and lead emergency responses in anesthesia-related work areas. S/he should assign tasks and should have clear communication channels to request appropriate help and ensure the integrity of anesthesia and related drug and equipment supplies, including adequate backup sources of oxygen.

- The anesthesiologist's priority must be to remain with the patient and take responsibility for their evacuation when structure collapse is imminent. Regard for personal safety is essential to ensure one's ability to provide care for those who are injured - including patients and staff alike.

- Anesthesia personnel should assume their designated tasks and collaborate with medical and support staff in other hospital areas.

- A key priority in response to an earthquake disaster is to place patients out of danger zones and into safe locations as quickly as possible.
- After the immediate event, longer-term planning to provide delivery of anesthesia services to damaged earthquake zones should be coordinated through a national health authority with input from a national society of anesthesiologists.

In our view, the inherent capacity for triage and for management of emergency situations and resources places anesthesiologists in a unique role when faced with the aftermath of an earthquake. Nevertheless, additional preparedness is essential when facing any sudden and unpredictable natural disaster. ${ }^{1-3}$

Conflicts of interest None declared.

Financial support None.

\section{References}

1. Chen $G$, Lai $W$, Liu $F$, et al. The dragon strikes: lessons from the Wenchuan earthquake. Anesth Analg 2010; 110: 908-15.

2. Rice MJ, Gwertzman A, Finley T, Morey TE. Anesthetic practice in Haiti after the 2010 Earthquake. Anesth Analg 2010; 111: 1445-9. 3. Lynch DK. Plan ahead. Anesth Analg 2010; 110: 653-4. 